\title{
1635 Inviscid real gas flow computations using kinetic scheme
}

\author{
P.S. Külkarni ${ }^{1}$, M. Sun ${ }^{1}$, T. saito ${ }^{2}$ and K. Takayama ${ }^{1}$
}

'Interdisciplinary Shock Wave Research Center, Institute of Fluid Science, Tohoku University, 2-1-1, Katahira, Aoba, Sendai 980-8577, Japan.Email: psk@rainbow.ifs.tohoku.ac.jp; psk@aero.iisc.ernet.in

${ }^{2}$ Muroran Institute of Technology, 27-1 Mizumoto-cho, Muroran, Hokkaido 050-8585, Japan

\section{ABSTRACT}

Kinetic Schemes over the years have become very useful and have been shown to be robust while computing inviscid flow for various types of bodies and for various flow regimes from subsonic to hypersonic flows. Further, algorithms based on meshless methods have shown to be more appropriate flow solvers for complex bodies. Re-entry vehicles enter the atmosphere at hypersonic speeds. The flow fields at theses hypersonic Mach numbers are chemically reacting, involving dissociation, recombination and possibly ionization of air molecules. The assumption of air as perfect gas at these Mach numbers is inaccurate and the real gas effects have to be taken into account to obtain realistic estimates of flow parameters. The objective of the present work is to incorporate real gas physics considering oxygen dissociation in the inviscid flow computations in the shock region to estimate flow parameters. The governing equations are modified assuming chemical equilibrium model.

\section{INTRODUCTION}

In Inviscid gas dynamics, Euler equations play a very important role as it can model many complex rotational flows of practical interest. Due to this fact many rescarchers have been engaged in solving complex flow problems over complex geometries using Euler equations. A large number of algorithms have been developed to solve inviscid compressible flows in order to increase the robustness, accuracy and reliability. Kinetic upwind methods (KFVS, PVU, and KNM) have their origin in kinetic theory of gases $[1,2]$. It is well known that the Euler equations are the moments of the Boltzmann equation when the velocity distribution function is Maxwellian. Varieties of flow problems over complex bodies have been solved using kinetic schemes and these algorithms are found to be robust [3]. However, grid generation is not only a non trivial issue for complex bodies but also plays very important role in accuracy of computations necessitating development of grid free algorithms: LSKUM (Least Square Kinetic Upwind method) is one such category for obtaining numerical solutions for Euler equations. In this paper an attempt has been made to develop a grid free flow solver based on kinetic scheme and further to incorporate the real gas physics in computations. Some preliminary investigations have been carried out considering 1D shock tube problem and the results are presented.

\section{KINETIC SCHEMES}

In kinetic schemes which are also known as Boltzmann schemes, Euler equations are obtained by: moment method strategy. Consider 1D Boltzmann equation $\cdots \ldots$,

$\frac{\partial f}{\partial t}+\mathrm{v} \frac{\partial f}{\partial x}=\mathbf{J}(\mathrm{f}, \mathrm{t})$
Where, $f=f(x, t, v)$ is the velocity distribution function and $v$ is the molecular velocity. The collision term $\mathbf{J}(\mathrm{f}, \mathrm{t})$ on the RHS of equation (1) vanishes when the velocity distribution is Maxwellian $\mathrm{F}$ in the Euler limit. $[3,4]$. Generally to get the correct $\gamma$, the ratio of specific heats, we need additional degree of freedom called I. which is a variable corresponding to internal degrees of freedom. Defining a moment function as

$\Psi=\left[1, v, I+\frac{\mathrm{v}^{2}}{2}\right]^{T}$

and then taking moments of Boltzmann equation leads to Euler equations given by.

$\frac{\partial U}{d t}+\frac{\partial G}{d x}=0 . \quad$ Where

$U=(\Psi, F)$ and $G=(\Psi, v F)$

$U=\left[\begin{array}{l}\rho \\ \rho u \\ \rho e\end{array}\right]$ and $G=\left[\begin{array}{l}\rho u \\ p+\rho u^{2} \\ (\rho e+p) u\end{array}\right]$

\section{LSKUM ALGORITHM}

This algorithm is basically a grid free method in the sense that is capable of operating on any arbitrary distribution of points. We describe briefly here the methodology.

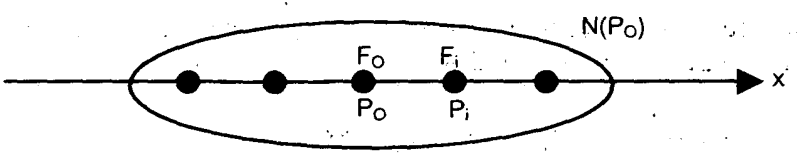

Fig 1. Least squares on arbitrary distribution of points in 1-D.

The basic idea behind LSKUM consists in obtaining a discrete approximation of derivative $\frac{\partial F}{\partial x}$ at a given point say $\mathbf{P}_{0}$ by using. least squares based on the surrounding neighbours. We have

$F_{0}=F_{1}+\Delta x_{i}\left(\frac{\partial F}{\partial x}\right)_{0}+\cdots i=1, n$. Where, 
$\Delta x_{i}=x_{i}-x_{0} ; \Delta F_{i}=F_{i}-F_{0}$ and $\mathrm{i}$ is the index of the grid point. We use the least square method which is based on minimizing the error

$\left\|e^{2}\right\|=\sum_{i=1}^{n}\left\{\Delta F_{i}-\Delta x_{i}\left(\frac{\Delta F}{\Delta x}\right)_{0}\right\}^{2}$, we then get a more general weighted least squares formula [ ] as follows.

$\left(\frac{\partial F}{d x}\right)_{0}=\frac{\sum_{i=1}^{n} w_{i} \Delta x_{i} \Delta F_{i}}{\sum_{i=1}^{n} w_{i} \Delta x_{i}^{2}}$

Where $w_{i}$ is the weight factor which can be appropriately chosen to construct upwind schemes $[4,5]$.

\section{REAL GAS EFFECTS}

The flow fields around the reentry vehicle at hypersonic Mach numbers are chemically reacting, involving dissociation, recombination and possibly ionization of air molecules. More realistic estimations of flow parameters can be obtained by taking real gas effects into account. In the present preliminary studies we have considered the dissociation of oxygen molecule in equilibrium conditions with air as working fluid. It is planned to take into account other reactions into account and also to extend the work to the multidimensional cases. Consider the dissociation reaction given by

$\mathrm{O}_{2}+\mathrm{O}_{2} \Leftrightarrow_{k_{-}}^{k^{+}} 2 \mathrm{O}+\mathrm{O}_{2}$

The energy e can be written as $e=h-\frac{p}{\rho}$, where $h=\alpha h_{o}+(1-\alpha) h_{o 2}$, o and $o_{2}$ are the atomic and molecular oxygen respectively. We have assumed the harmonic oscillator model for the vibrational energy while computing $c_{v}$ the specific heat at constant volume. The equilibrium constant $\mathrm{K}$ is related to the other parameters by the following relation using which $\alpha$ can be determined.

$\frac{4 \alpha^{2}}{1-\alpha^{2}}=\frac{K R T}{p}$

The equilibrium constants K's are exponential functions of temperature and are expressed as

$K(T)=\operatorname{Exp}\left[c_{0}+c_{1} T_{1}+c_{2} T_{2}+c_{3} T_{3}+c_{4} T_{4}\right]$

Where $\quad T_{1}=10000 / T, T_{2}=T_{1} \times T_{1}, T_{3}=T_{2} \times T_{1}, T_{4}=T_{3} \times T_{1}$ and

$c_{0}=1.335, c_{1}=-4.127, c_{2}=-0.616, c_{3}=0.093, c_{4}=-0.005$

\section{RESULTS AND DISCUSSIONS}

With these modifications the LSKUM code has been run for shock tube problem with various pressure ratios like $25,50,100$ and 150 which cover hypersonic flow regime as shown in figures 2 to 4 . For lower pressure ratios we find not much difference in the perfect gas and equilibrium model cases. However, for higher Mach numbers we find an appreciable difference in the temperature behind the shock wave as expected. The details of analysis and additional results will be presented. Grid free method offers many advantages over other methods while computing flow over complex bodies. Extension of the present study to higher dimension cases is being carried out.

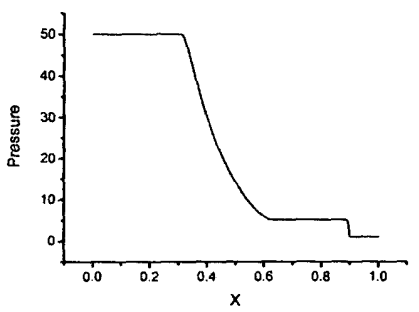

Fig.2 Pressure ratio of 1:50

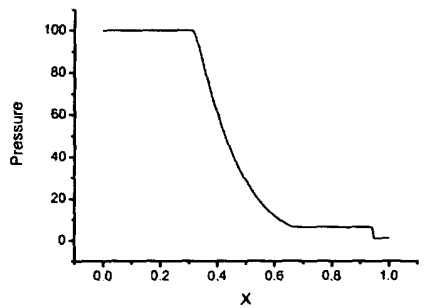

Fig. 3 Pressure ratio of 1:100

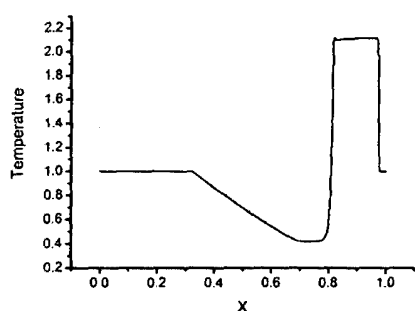

Fig. 4. Pressure ratio of $1: 150$

\section{REFERENCES}

1. Pullin D.I., Direct Simulation methods for compressible inviscid ideal gas flows, Jl. Computational physics, 34(2),231-244, (1980).

2. Deshpande S.M., On the Maxwellian distribution, symmetric form, and entropy conservation for Euler equations, NASA-TP2583 (1986)

3. Sekar S, M Nagarathinam, Krishnamurthy R., Kulkarni P.S. and Deshpande S.M., 3-D KFVS Euler Code "BHEEMA" as aerodynamic design and analysis tool for Complex Configurations, Proceedings $14^{\text {th }}$ ICNMFD, Bangalore, July 11-15, 1994, Lecture Notes in Physics No. 453, pp 525-529.

4. Ghosh A.K. and Deshpande S.M., Least squares kinetic upwind method for compressible flows, AIAA paper no. 95-1735

5. Deshpande S.M., Kulkarni PS, Ghosh,A.K., New Developments in Kinetic Schemes, JI. Of Computers and mathematics with Applications, vol 35, No. 1/2, pp 75-93, (1998). 